\title{
Deep Ice and DNA languages
}

\section{Life, but not intelligence, abounds under the ice of frozen planets.}

\section{Greg Bear}

2215 CE - The discovery of more than 15,000 Massive Deep-Ice Objects (MDIOs) in orbit around supermassive planets in the close interstellar neighbourhood has the potential to revolutionize our understanding of biological languages.

Phenotype-generating languages for terrestrial DNA-recorded life forms can be ranked closely according to kingdoms. Most plants, for example, express phylogeny according to the famous Zinn-Wang languages, first decoded in the mid-twenty-first century.

Archaea, commonly used as the Rosetta Stone for all primitive DNA languages, have provided deep insights into nonterrestrial biologies that have advanced to the DNA level.

RNA languages in terrestrial viruses constitute a virtual Tower of Babel, indicating a degenerate and mutationally rich mix that can still compel replication in DNA hosts. Early RNA coding systems found outside the Solar System, however, can often be translated into Archaean DNA-based languages, and these may constitute the most basic fixed languages of life.

MDIOs are typically seven to nine Earth masses and consist of layers of water ice, $2,000-5,000 \mathrm{~km}$ in depth, overlying a highpressure liquid ocean (HPLO) that sits in turn on a rocky mantle. At the centre is an iron- and sulphur-rich core.

The interior is warmed both by latent heat and radioactivity (chiefly thorium-90) and by tidal friction generated by interaction with the parent supermassive body. Temperatures in the HPLO can frequently exceed $130{ }^{\circ} \mathrm{C}$. MDIOs may constitute the most common life-supporting bodies in the Universe.

Neutronium self-guided probes (NSGP) have penetrated seven of these 'deep ice' objects and have obtained remarkable data. Spin-off probes made of normal matter, transmuted from neutronium and released into the HPLO, perform in situ analysis of all carbon chemistry and send information to orbiting research stations.

What is most remarkable about MDIO biologies is that they can exist at all under these extraordinary conditions. Once again, it is demonstrated that life will begin and thrive anywhere there is liquid water and the necessary elements.

To date, RNA- and DNA-language analysis has been conducted on three HPLO ecosystems. Because of limited exploration

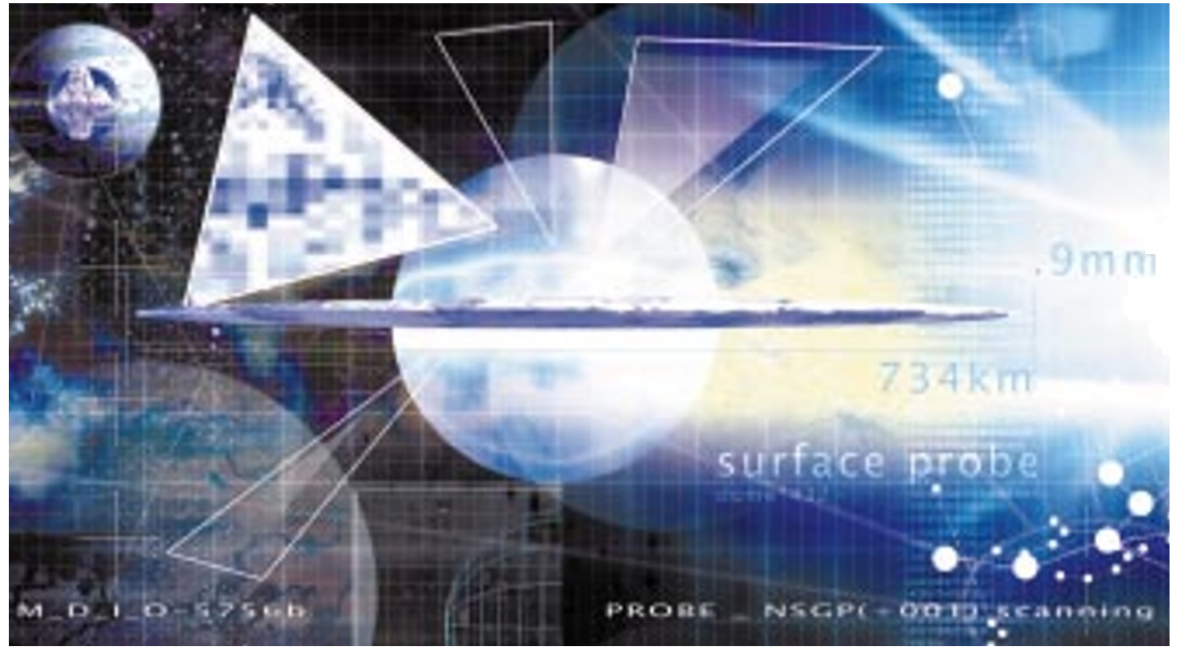

ranges for the probes, the extent of these ecosystems is not known: however, every probed MDIO possesses life.

One of the ecosystems (MDIO 2341a) is still in a 'profligate', mutation-rich RNA phase, with no complex organisms and no DNA detected. Here, new genetic coding schemes may naturally emerge every few months, and competition between coding schemes is likely to be extreme. (The emergence of competent and stable genetic languages on Earth may have taken more than a billion years.)

The other two ecosystems (MDIO 5756b and MDIO 349x) have entered the more stable age of DNA and show remarkable similarities to each other.

The most striking feature of these ecosystems is how bright they are, since they are completely hidden from all starlight. The roving probes have sent back images of massive reef-like structures glowing as brightly as several full moons, surrounded by a thick, slowly churning mass of light-dependent microbes. Feeding on these microbes are living filter nets, fringed by corkscrew cilia, able to join into larger units or separate into smaller ones.

Tall spiral chimneys, like baroque columns in a church, release water heated in the upper mantle, creating plumes that can extend for many kilometres. These plumes spread out at the upper ice layer, eroding smooth domes almost a hundred kilometres in diameter and usually less than a millimetre deep. These domes collect oxygen freed from the actions of photosynthetic organisms. Typically the oxygen is forced back under extreme pressure into the water and the ice within seconds, but during this brief time, miniature forests of opportunis- tic organisms grow in the domes, extracting all the benefits from a more efficient oxygen metabolism.

The upper limit of organization in MDIO ecosystems is not known. Typical distributed-intelligence ecosystems are found here, and interact to form complex neuronal networks that govern MDIO life cycles (as on Earth). However, no condensed nodal intelligences such as large animals have yet been found. Instead, intelligence seems frozen at a very early and distributed stage.

This may reflect the unlikelihood of any intelligences within the MDIO ever being challenged by major environmental change, much less being given a chance to observe the outside Universe. (MDIOs seem to be remarkably stable over hundreds of millions of years.)

The impossibility of emergence through the deep ice and escape into space limits the potential growth of concentrated hypothesis engines as defined by the Turing-Watteau diagram of novel information versus expansion opportunity.

Some researchers suggest that the seeding of provocative artefacts ('Clarkeing') below the deep ice may encourage condensation of concentrated intelligences, or, at the very least, induce some interesting emergent properties. The design of these artefacts is currently spurring intense debate. As one chief communications researcher has asked, "How do you uplift slime?"

Harnessing of bacterial communities on Earth in the last century could provide a template for working with MDIO ecosystems, adding to the list of beings we can actually talk to.

Greg Bear is the author of 26 novels, the most recent being Darwin's Radio (HarperCollins). 